\title{
Human Epidermal Growth Factor Receptor 2-positive Primary Adenocarcinoma in the Cervical Oesophagus: A Case Report
}

\author{
TOMO HORINOUCHI, NAOYA YOSHIDA, CHIHIRO MATSUMOTO, YOSHIHIRO HARA, \\ TASUKU TOIHATA, MASAAKI IWATSUKI, YOSHIFUMI BABA, YUJI MIYAMOTO and HIDEO BABA \\ Department of Gastroenterological Surgery, Graduate School of Medical Sciences, \\ Kumamoto University, Kumamoto, Japan
}

\begin{abstract}
Background/Aim: Cervical oesophageal adenocarcinoma (COA) is extremely rare. We present a case of human epidermal growth factor receptor 2 (HER2)-positive COA that showed repeated recurrences despite multidisciplinary treatments. Case Report: A 49-year-old male was diagnosed with clinical stage IVA COA that originated from ectopic gastric mucosa. He initially underwent definitive chemoradiotherapy (CRT) (60.0 Gy/30 fractions, 5fluorouracil, and cisplatin). Two months after CRT, the right supraclavicular lymph node (LN) reenlarged and salvage lymphadenectomy was performed. Immunohistochemical staining revealed a HER2-positive adenocarcinoma. Four months after lymphadenectomy, multiple metastases in the mediastinal LNs and lungs were detected, and S-1, oxaliplatin and trastuzumab were administered. Four months after chemotherapy, the patient presented with new liver metastasis. Further metastasis was prevented by Nivolumab treatment for four months. Conclusion: HER2-positive COA may be more aggressive and may require further intensive treatments. This literature review may be helpful in determining treatment strategies for $C O A$.
\end{abstract}

The main histological types of oesophageal cancer are squamous cell carcinoma (SCC) and adenocarcinoma (1). Oesophageal adenocarcinoma generally occurs in the lower oesophagus and arises from Barrett's epithelium associated with reflux oesophagitis (1). However, oesophageal

This article is freely accessible online.

Correspondence to: Naoya Yoshida, MD, Ph.D., Department of Gastroenterological Surgery, Graduate School of Medical Sciences, Kumamoto University, 1-1-1 Honjo, Chuoku, Kumamoto 860-8556, Japan. Tel: +81 963735212, Fax: +81 963714378, e-mail: nyoshida@kumamoto-u.ac.jp

Key Words: Oesophageal adenocarcinoma, cervical oesophagus, HER2. adenocarcinoma in the cervical oesophagus is extremely rare. Thus, a treatment strategy has not been established. Here, we present a case of human epidermal growth factor receptor 2 (HER2)-positive cervical oesophageal adenocarcinoma that showed repeated recurrences despite multidisciplinary treatments. In addition, we review reported cases to develop a treatment strategy for this rare form of cancer.

\section{Case Report}

A 49-year-old male visited a clinic due to sore throat. Esophagoscopy revealed a tumour in the cervical oesophagus, and he was referred to our hospital for scrutiny and subsequent treatment. He had no history of major illness or family history of cancer. He had inveterate habits of drinking seven bottles of beer per week and smoking 1 packper-day for 29 years.

Esophagoscopy revealed a half-circumferential tumour at the left wall in the cervical oesophagus, $17-25 \mathrm{~cm}$ from the incisors (Figure 1A). Histopathological examination of the biopsy samples showed adenocarcinoma (Figure 1B). Computed tomography (CT) suggested a tumour in the cervical oesophagus that may invade the trachea and metastases to the right supraclavicular and right recurrent laryngeal nerve lymph nodes (LNs) (Figure 1C and D) but no distant metastases. Fluorodeoxyglucose (FDG) positron emission tomography (PET)/CT showed high FDG uptake with maximum standardized uptake values $\left(\mathrm{SUV}_{\max }\right)$ of 34.6, 11.9, and 6.8 in the primary lesion, right supraclavicular LN, and right recurrent laryngeal nerve LN, respectively (Figure 1E and $\mathrm{F}$ ). Oesophagography revealed a wall irregularity in the cervical oesophagus (Figure 1G). Finally, the tumour was diagnosed as clinical T4b, N2, M0, stage IVA adenocarcinoma in the cervical oesophagus [Union for International Cancer Control (UICC) $8^{\text {th }} \mathrm{Ed}$ ].

The patient underwent definitive chemoradiotherapy (CRT) consisting of a total radiation dose of $60.0 \mathrm{~Gy} / 30$ fractions for the primary lesion and LN metastasis, and 40.0 $\mathrm{Gr} / 20$ fractions for the regional LNs, and concurrent 

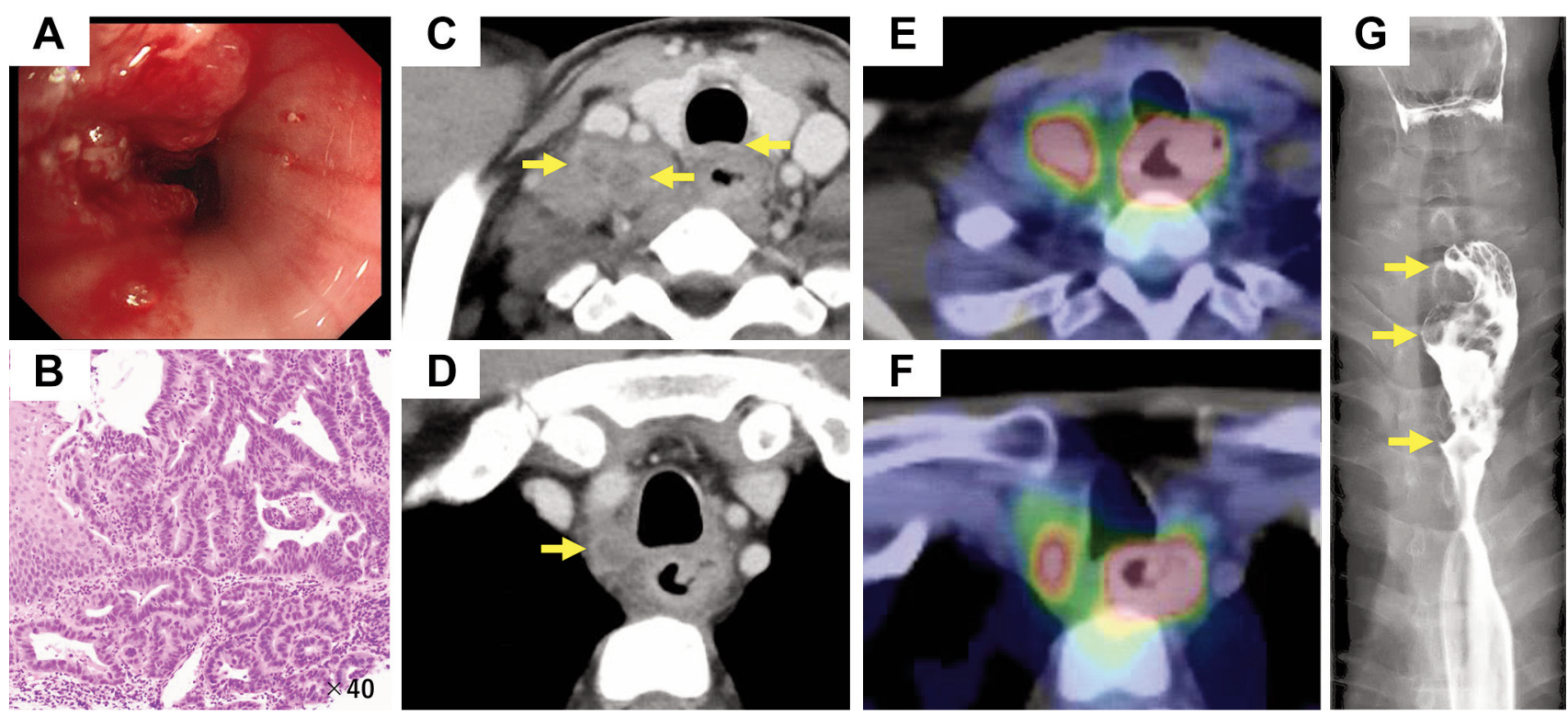

Figure 1. Preliminary evaluations. Esophagoscopy shows a half-circumferential tumour at the left wall of the cervical oesophagus, 17-25 cm from the incisors (A). Histopathological examination of biopsy samples shows adenocarcinoma (B). Computed tomography (CT) suggests a tumour in the cervical oesophagus that is likely to invade the trachea, and metastases to the right supraclavicular and right recurrent laryngeal nerve lymph nodes (LNS) (C and D, yellow arrowheads). Fluorodeoxyglucose (FDG) positron emission tomography/computed tomography/CT shows high FDG uptake with a maximum standardized uptake value $\left(S U V_{\max }\right)$ of 34.6, 11.9, and 6.8 in the primary lesion, right supraclavicular $L N$, and right recurrent laryngeal nerve $L N$, respectively $(E$ and $F)$. Oesophagography reveals a wall irregularity in the cervical oesophagus ( $G$, yellow arrowheads).

administration of 5-fluorouracil and cisplatin $(\mathrm{FP})$ regimen chemotherapy. The FP regimen consisted of cisplatin 80 $\mathrm{mg} / \mathrm{m}^{2} /$ day, administered intravenously (IV) on day 1 , followed by 5 -fluorouracil $800 \mathrm{mg} / \mathrm{m}^{2} /$ day, administered continuously IV during the period from day 1 to day 5. Two courses were administered at 4-week intervals during CRT. PET/CT two weeks after CRT showed a remarkable decrease in FDG accumulation in both the primary tumour and metastatic LNs. The patient underwent two additional courses of FP regimen chemotherapy. However, PET/CT two months after CRT showed a re-enlargement of the right supraclavicular LN with FDG accumulation with an $\mathrm{SUV}_{\max }$ of 11.2 (Figure 2A). Thus, salvage lymphadenectomy was performed as a curative measure. Immunohistochemical staining of the resected LN revealed a HER2 (3+) oesophageal adenocarcinoma metastasis (Figure 2B and C).

Four months after salvage lymphadenectomy, multiple novel metastases were detected on $\mathrm{CT}$ in the mediastinal LNs and lungs (Figure 3). Based on the pathological HER-2 status, the patient underwent $\mathrm{S}-1$ and oxaliplatin (SOX) regimen chemotherapy plus trastuzumab as the second regimen (2). Four months after the chemotherapy, new liver metastasis appeared and Nivolumab was administered as the third regimen. The metastasis did not worsen, and the patient survived for 4 months after Nivolumab treatment.

\section{Discussion}

In Japan, SCC has long been the dominant histological type of oesophageal cancer, representing over $85 \%$ of these cancers (3). However, in recent years, the prevalence of oesophageal adenocarcinoma has increased rapidly from $1.7 \%$ to $10 \%$ (4) as the prevalence of reflux esophagitis increased, possibly due to the increase in obesity. Meanwhile, oesophageal adenocarcinoma in the cervical oesophagus is extremely rare. To the best of our knowledge, only 24 cases of adenocarcinoma in the cervical oesophagus, including our case, have been reported (Table I). Based on our review of this literature, the median age of the patients was 65.5 years, and $83.3 \%$ were male. Most of the cases were ectopic and originated from the gastric mucosa, and only $16.7 \%$ were associated with extensive Barrett's oesophagus. Our case did not have extensive Barrett's oesophagus, suggesting that it was ectopic and originated from the gastric mucosa in the cervical oesophagus.

Treatment strategies for cervical oesophageal adenocarcinoma have not been established due to the rarity of this condition. In the present case, CRT with the FP regimen was initially administered to preserve the voice, and the tumour seemed to disappear temporally. However, LN metastasis was revealed only 2 months after CRT. Although salvage 

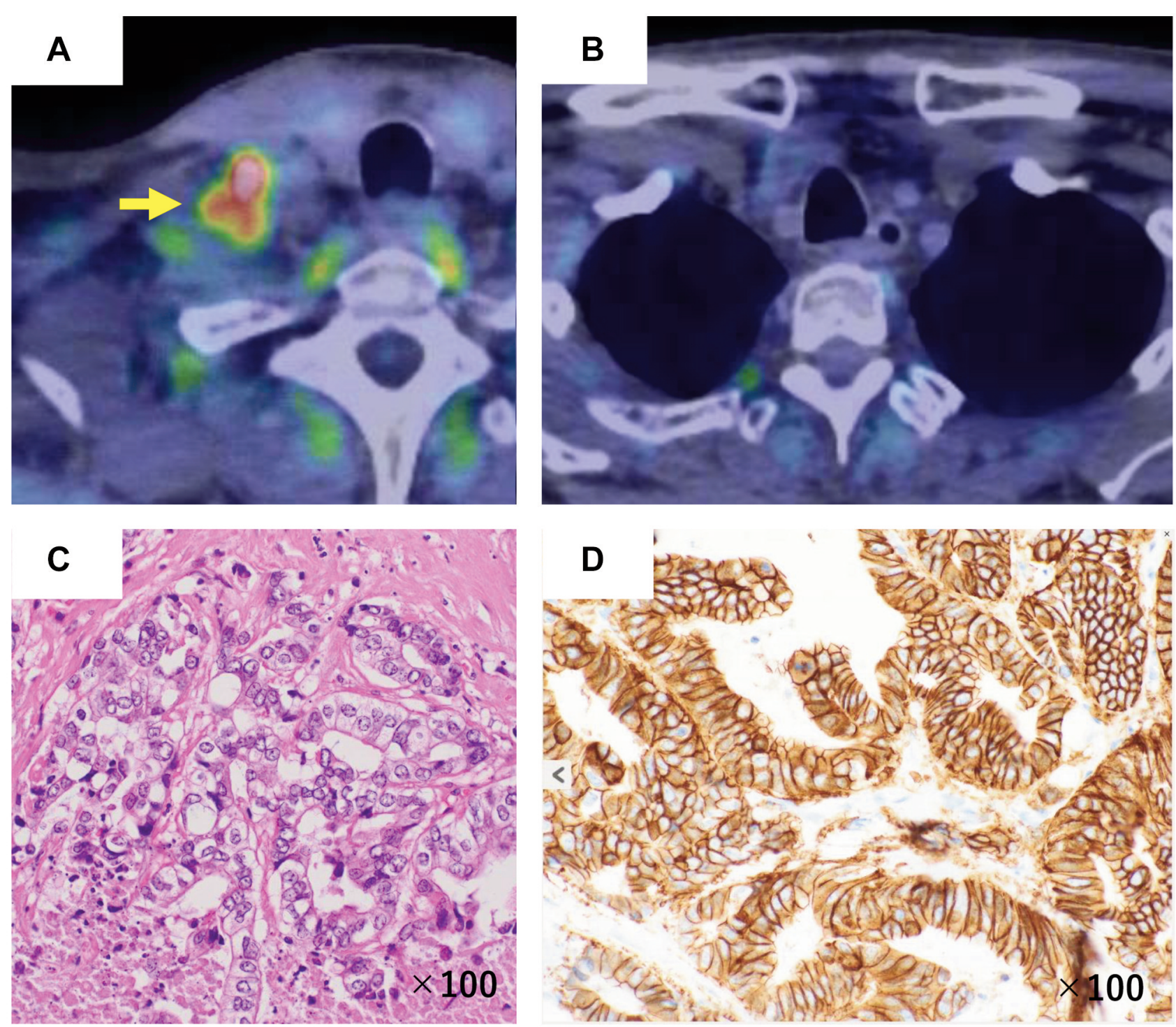

Figure 2. Post-treatment evaluations. Positron emission tomography/computed tomography (PET/CT) two months after chemoradiotherapy shows re-enlargement of the right supraclavicular lymph node with fluorodeoxyglucose accumulation and a maximum standardized uptake value of 11.2 (A, yellow arrowhead). There are no- enlargement of the cervical oesophagus and right recurrent laryngeal nerve lymph nodes (B). Immunohistochemical staining after salvage lymphadenectomy indicates HER2-positive (3+) oesophageal adenocarcinoma metastasis (C and D).

lymphadenectomy and adjuvant chemotherapy were performed for cure, multiple metastases occurred shortly after the treatments. Immunohistochemistry revealed that the recurrent lesions were HER2-positive, predicting a cancer with a highly malignant potential and a high risk of recurrence (5). Our case suggested that cervical adenocarcinoma with a positive HER2 status may be more aggressive than that with a negative HER2 status even in cervical oesophageal adenocarcinoma. Notably, subsequent chemotherapy with SOX and trastuzumab could not prevent further progression, and treatment with an immunecheckpoint inhibitor is ongoing.
The literature review may help determine a treatment strategy $(4,6-25)$. Most of the reported patients with stage 0 and I adenocarcinomas underwent endoscopic treatment or surgery and had considerably good results without any recurrences $(4,8,11,12,14,20-25)$. For patients with stage II, surgery alone without adjuvant treatment was adequate $(9$, 18, 22). In contrast, patients with stage III and higher advanced cancer underwent various treatments, including CRT and surgery $(10,13,15,16,20)$. However, recurrence was observed in $43 \%$ of these patients, and the median survival was only 19 months, which suggested that further 


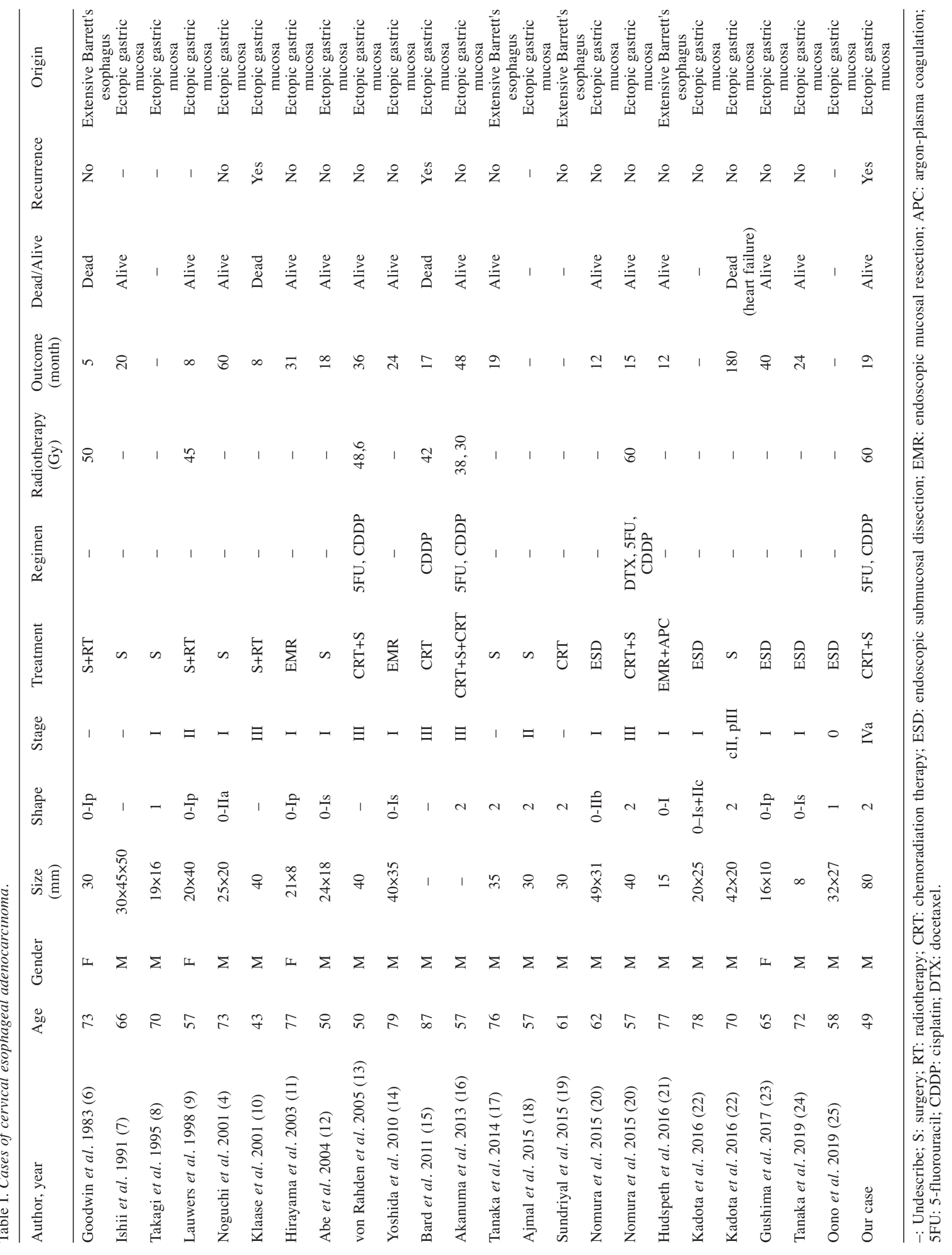



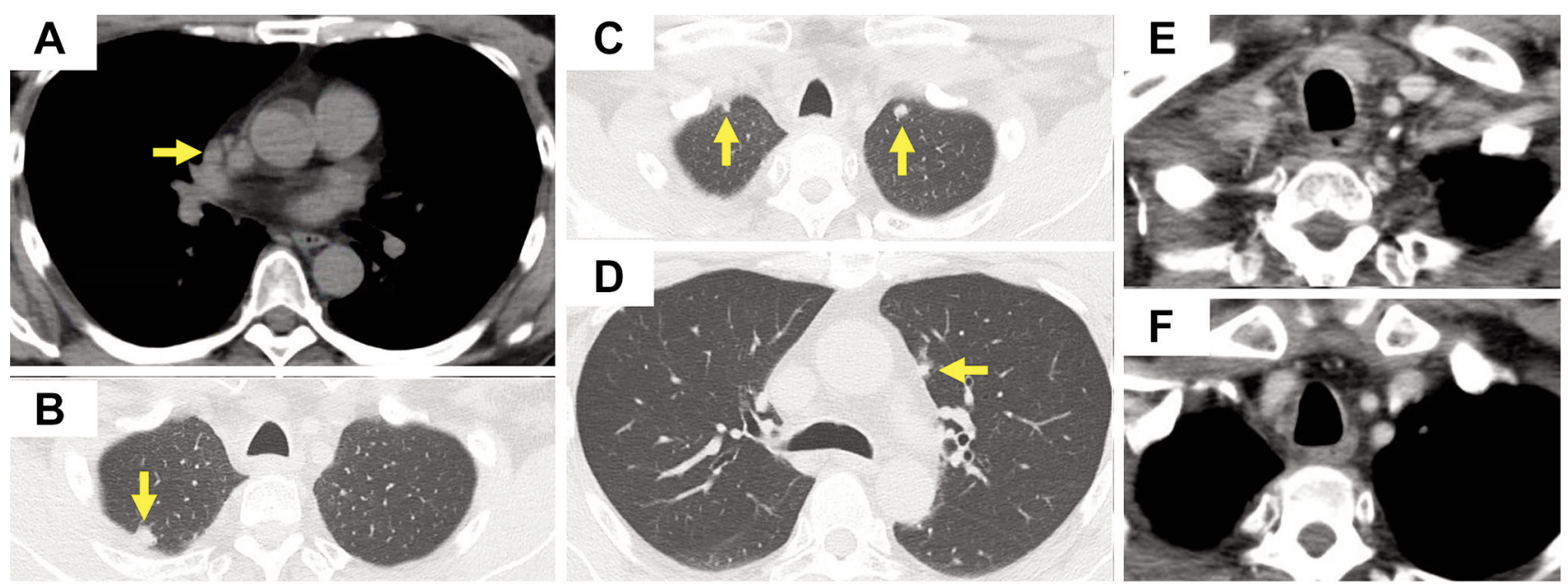

Figure 3. Computed tomography four months after salvage lymphadenectomy. Multiple new metastases are seen in the mediastinal lymph nodes (A, yellow arrowhead) and the lungs ( $B, C$ and $D$, yellow arrowheads). There are no tumours in the cervical oesophagus, and no metastases to the right supraclavicular and right recurrent laryngeal nerve $L N s$ ( $E$ and $F$ ).

advances in the treatment strategy are necessary for advanced cervical oesophageal adenocarcinoma.

To our knowledge, this is the first report of a case of HER2-positive primary cervical oesophageal adenocarcinoma. Our experience suggests that a HER2positive tumors have a highly malignant potential, and further intensive treatments are recommended. Recently, a novel regimen with trastuzumab deruxtecan was reported to improve survival of patients with HER2-positive gastric cancer (26). In addition, novel agents such as immunecheckpoint inhibitors and multi-kinase inhibitors have proven effective against gastric and esophagogastric adenocarcinoma (27-29). Further progress in treatment strategies, including those agents, is necessary to improve the treatment outcomes of advanced adenocarcinoma in the cervical oesophagus.

\section{Conclusion}

Our case suggests the existence of an aggressive cervical oesophageal adenocarcinoma. It is necessary to accumulate the data on treatment outcomes and progress treatment strategy for cervical oesophageal adenocarcinoma to further improve survival outcome.

\section{Conflicts of Interest}

The Authors declare no conflicts of interest in relation to this study.

\section{Authors' Contributions}

TH described and designed the article. NY edited the article. HB supervised the editing of the manuscript. NY, MI and YB were involved in the operation of the patient. $\mathrm{CM}, \mathrm{YH}$ and TT collected the data. All Authors read and approved the final manuscript.

\section{Acknowledgements}

The Authors would like to thank Editage (www.editage.com) for English language editing.

\section{References}

1 Pennathur A, Gibson MK, Jobe BA and Luketich JD: Oesophageal carcinoma. Lancet 381(9864): 400-412, 2013. PMID: 23374478. DOI: 10.1016/S0140-6736(12)60643-6

2 Bang YJ, Van Cutsem E, Feyereislova A, Chung HC, Shen L, Sawaki A, Lordick F, Ohtsu A, Omuro Y, Satoh T, Aprile G, Kulikov E, Hill J, Lehle M, Rüschoff J, Kang YK and ToGA Trial Investigators: Trastuzumab in combination with chemotherapy versus chemotherapy alone for treatment of HER2-positive advanced gastric or gastro-oesophageal junction cancer (ToGA): a phase 3, open-label, randomised controlled trial. Lancet 376(9742): 687-697, 2010. PMID: 20728210. DOI: 10.1016/S0140-6736(10)61121-X

3 Watanabe M, Tachimori Y, Oyama T, Toh Y, Matsubara H, Ueno M, Kono K, Uno T, Ishihara R, Muro K, Numasaki H, Tanaka K, Ozawa S, Murakami K, Usune S, Takahashi A, Miyata H and Registration Committee for Esophageal Cancer of the Japan Esophageal Society: Comprehensive registry of esophageal cancer in Japan, 2013. Esophagus 18(1): 1-24, 2021. PMID: 33047261. DOI: 10.1007/s10388-020-00785-y

4 Noguchi T, Takeno S, Takahashi Y, Sato T, Uchida Y and Yokoyama S: Primary adenocarcinoma of the cervical esophagus arising from heterotopic gastric mucosa. J Gastroenterol 36(10): 704-709, 2001. PMID: 11686482. DOI: 10.1007/s005350170035

5 Lei YY, Huang JY, Zhao QR, Jiang N, Xu HM, Wang ZN, Li HQ, Zhang SB and Sun Z: The clinicopathological parameters and prognostic significance of HER2 expression in gastric 
cancer patients: a meta-analysis of literature. World J Surg Oncol 15(1): 68, 2017. PMID: 28327158. DOI: 10.1186/s12957-017$1132-5$

6 Goodwin WJ Jr, Larson DL and Sajjad SM: Adenocarcinoma of the cervical esophagus in a patient with extensive columnar celllined (Barrett's) esophagus. Otolaryngol Head Neck Surg 91(4): 446-449, 1983. PMID: 6415597. DOI: 10.1177/ 019459988309100420

7 Ishii K, Ota H, Nakayama J, Katsuyama T, Matsuzawa K, Honda $\mathrm{T}$ and Akamatsu T: Adenocarcinoma of the cervical oesophagus arising from ectopic gastric mucosa. The histochemical determination of its origin. Virchows Arch A Pathol Anat Histopathol 419(2): 159-164, 1991. PMID: 1871960. DOI: 10.1007/BF01600231

8 Takagi A, Ema Y, Horii S, Morishita M, Miyaishi O and Kino I: Early adenocarcinoma arising from ectopic gastric mucosa in the cervical esophagus. Gastrointest Endosc 41(2): 167-170, 1995. PMID: 7721010. DOI: 10.1016/s0016-5107(05)80605-4

9 Lauwers GY, Scott GV and Vauthey JN: Adenocarcinoma of the upper esophagus arising in cervical ectopic gastric mucosa: rare evidence of malignant potential of so-called "inlet patch". Dig Dis Sci 43(4): 901-907, 1998. PMID: 9558051. DOI: 10.1023/ a:1018855223225

10 Klaase JM, Lemaire LC, Rauws EA, Offerhaus GJ and van Lanschot JJ: Heterotopic gastric mucosa of the cervical esophagus: a case of high-grade dysplasia treated with argon plasma coagulation and a case of adenocarcinoma. Gastrointest Endosc 53(1): 101-104, 2001. PMID: 11154502. DOI: 10.1067/ mge.2001.111394

11 Hirayama N, Arima M, Miyazaki S, Shimada H, Okazumi S, Matsubara H, Suzuki T, Ochiai T and Kouzu T: Endoscopic mucosal resection of adenocarcinoma arising in ectopic gastric mucosa in the cervical esophagus: case report. Gastrointest Endosc 57(2): 263-266, 2003. PMID: 12556801. DOI: 10.1067/ mge. 2003.84

12 Abe T, Hosokawa M, Kusumi T, Kusano M, Hokari K, Kagaya $\mathrm{H}$, Watanabe A, Fujita M and Sasaki S: Adenocarcinoma arising from ectopic gastric mucosa in the cervical esophagus. Am J Clin Oncol 27(6): 644-645, 2004. PMID: 15577449. DOI: 10.1097/01.coc.0000147808.63442.b5

13 von Rahden BH, Stein HJ, Becker $K$ and Siewert RJ: Esophageal adenocarcinomas in heterotopic gastric mucosa: review and report of a case with complete response to neoadjuvant radiochemotherapy. Dig Surg 22(1-2): 107-112, 2005. PMID: 15849472 . DOI: $10.1159 / 000085448$

14 Yoshida T, Shimizu Y and Kato M: Image of the month. Use of magnifying endoscopy to identify early esophageal adenocarcinoma in ectopic gastric mucosa of the cervical esophagus. Clin Gastroenterol Hepatol 8(9): e91-e93, 2010. PMID: 20347051. DOI: 10.1016/j.cgh.2010.02.025

15 Bard A, Coton T, De Biasi C and Guisset M: Adenocarcinoma of the upper esophagus. Clin Res Hepatol Gastroenterol 35(5): 418-419, 2011. PMID: 21354890. DOI: 10.1016/j.clinre. 2011.01 .010

16 Akanuma N, Hoshino I, Akutsu Y, Shuto K, Shiratori T, Kono T, Uesato M, Sato A, Isozaki Y, Maruyama T, Takeshita N and Matsubara H: Primary esophageal adenocarcinoma arising from heterotopic gastric mucosa: report of a case. Surg Today 43(4): 446-451, 2013. PMID: 22706784. DOI: 10.1007/s00595-0120206-9
17 Tanaka M, Ushiku T, Ikemura M, Shibahara J, Seto Y and Fukayama M: Esophageal adenocarcinoma arising in cervical inlet patch with synchronous Barrett's esophagus-related dysplasia. Pathol Int 64(8): 397-401, 2014. PMID: 25143128. DOI: $10.1111 /$ pin.12181

18 Ajmal S, Young JS and Ng T: Adenocarcinoma arising from cervical esophageal gastric inlet patch. J Thorac Cardiovasc Surg 149(6): 1664-1665, 2015. PMID: 25911182. DOI: 10.1016/ j.jtcvs.2015.03.050

19 Sundriyal D, Shirsi N, Kotwal S, Kumar S, Mithran Parthasarthy $\mathrm{K}$ and Gupta M: Adenocarcinoma of Cervical Esophagus Arising in the Barrett's Mucosa. Indian J Surg Oncol 6(2): 127-129, 2015. PMID: 26405420. DOI: 10.1007/s13193-015-0382-3

20 Nomura K, Iizuka T, Inoshita N, Kuribayashi Y, Toba T, Yamada A, Yamashita S, Furuhata T, Kikuchi D, Matsui A, Mitani T, Ogawa O, Hoteya S, Ueno M, Udagawa $\mathrm{H}$ and Kaise $\mathrm{M}$ : Adenocarcinoma of the cervical esophagus arising from ectopic gastric mucosa: report of two cases and review of the literature. Clin J Gastroenterol 8(6): 367-376, 2015. PMID: 26476962. DOI: $10.1007 / \mathrm{s} 12328-015-0610-\mathrm{y}$

21 Hudspeth VR, Smith DS, Pacicco T and Lewis JJ: Successful endoscopic resection of adenocarcinoma arising in an esophageal inlet patch. Dis Esophagus 29(7): 880-882, 2016. PMID: 24961886. DOI: 10.1111/dote.12249

22 Kadota T, Fujii S, Oono Y, Imajoh M, Yano T and Kaneko K: Adenocarcinoma arising from heterotopic gastric mucosa in the cervical esophagus and upper thoracic esophagus: two case reports and literature review. Expert Rev Gastroenterol Hepatol 10(3): 405-414, 2016. PMID: 26610162. DOI: 10.1586/ 17474124.2016.1125780

23 Gushima R, Narita R, Shono T, Naoe H, Yao T and Sasaki Y: Esophageal adenocarcinoma with enteroblastic differentiation arising in ectopic gastric mucosa in the cervical esophagus: a case report and literature review. J Gastrointestin Liver Dis 26(2): 193-197, 2017. PMID: 28617891. DOI: 10.15403/ jgld.2014.1121.262.gus

24 Tanaka K, Yamada R, Tsuboi J, Kuroda N and Hamada Y: A small adenocarcinoma in the cervical esophagus. VideoGIE 4(3): 111-113, 2019. PMID: 30899886. DOI: 10.1016/j.vgie. 2018.12 .003

25 Oono Y, Kensuke S, Yoda Y, Hori K, Ikematsu H and Yano T: Cervical esophageal adenocarcinoma arising from heterotopic gastric mucosa, treated with endoscopic submucosal dissection. Endoscopy 51(2): E28-E29, 2019. PMID: 30469153. DOI: 10.1055/a-0767-6253

26 Shitara K, Bang YJ, Iwasa S, Sugimoto N, Ryu MH, Sakai D, Chung HC, Kawakami H, Yabusaki H, Lee J, Saito K, Kawaguchi Y, Kamio T, Kojima A, Sugihara M, Yamaguchi K and DESTINY-Gastric01 Investigators: Trastuzumab deruxtecan in previously treated HER2-positive gastric cancer. N Engl J Med 382(25): 2419-2430, 2020. PMID: 32469182. DOI: 10.1056/NEJMoa2004413

27 Wilke H, Muro K, Van Cutsem E, Oh SC, Bodoky G, Shimada Y, Hironaka S, Sugimoto N, Lipatov O, Kim TY, Cunningham D, Rougier P, Komatsu Y, Ajani J, Emig M, Carlesi R, Ferry D, Chandrawansa K, Schwartz JD, Ohtsu A and RAINBOW Study Group: Ramucirumab plus paclitaxel versus placebo plus paclitaxel in patients with previously treated advanced gastric or gastro-oesophageal junction adenocarcinoma (RAINBOW): a double-blind, randomised phase 3 trial. Lancet Oncol 15(11): 
1224-1235, 2014. PMID: 25240821. DOI: 10.1016/S14702045(14)70420-6

28 Kang YK, Boku N, Satoh T, Ryu MH, Chao Y, Kato K, Chung HC, Chen JS, Muro K, Kang WK, Yeh KH, Yoshikawa T, Oh SC, Bai LY, Tamura T, Lee KW, Hamamoto Y, Kim JG, Chin K, Oh DY, Minashi K, Cho JY, Tsuda M and Chen LT: Nivolumab in patients with advanced gastric or gastro-oesophageal junction cancer refractory to, or intolerant of, at least two previous chemotherapy regimens (ONO-4538-12, ATTRACTION-2): a randomised, double-blind, placebo-controlled, phase 3 trial. Lancet 390(10111): 2461-2471, 2017. PMID: 28993052. DOI: 10.1016/S0140-6736(17)31827-5

29 Shitara K, Özgüroğlu M, Bang YJ, Di Bartolomeo M, Mandalà M, Ryu MH, Fornaro L, Olesiński T, Caglevic C, Chung HC,
Muro K, Goekkurt E, Mansoor W, McDermott RS, ShachamShmueli E, Chen X, Mayo C, Kang SP, Ohtsu A, Fuchs CS and KEYNOTE-061 investigators: Pembrolizumab versus paclitaxel for previously treated, advanced gastric or gastro-oesophageal junction cancer (KEYNOTE-061): a randomised, open-label, controlled, phase 3 trial. Lancet 392(10142): 123-133, 2018. PMID: 29880231. DOI: 10.1016/S0140-6736(18)31257-1

Received April 20, 2021 Revised April 30, 2021 Accepted May 4, 2021 